\title{
An Application of Peer Review and Project- Based Learning with the Aim of Boost Students' Employability
}

\author{
Cristian Martín ${ }^{1 *}$, Antonio Muñoz ${ }^{1}$, Alicia Tocino ${ }^{2}$, Rafael Moreno-Sáez $^{3}$ and Sergio \\ Fortes $^{4}$ \\ ${ }^{1}$ Computer Science Department, University of Malaga, Campus de Teatinos s/n, Malaga, Spain \\ ${ }^{2}$ Algebra, Geometry and Topology Department, Faculty of Sciences, University of Malaga, Campus de \\ Teatinos s/n, Málaga, Spain \\ ${ }^{3}$ Electronic Technology Department, University of Málaga, Spain \\ ${ }^{4}$ Communications Engineering Department, University of Málaga, Andalucía Tech, Spain \\ *Corresponding author
}

\begin{abstract}
Students often lack sufficient experience to deal with real problems at the beginning of their working life. In this article, we present the application of an approach based on project-based learning and peer review to incentive this process. This approach allows, on the one hand, to bring students closer to real problems of society, and on the other hand, to raise their critical spirit while encouraging their motivation. Moreover, peer review also allows students to learn about other approaches to solving a problem, which enhances and enriches their learning process. This experience has been applied in an IT course at the University of Malaga, where 81 students had to design and develop an Android application solving a social problem. The results obtained show an improvement in student performance compared to previous academic years.
\end{abstract}

Keywords: peer review, students' employability, project-based learning

\section{Introduction}

Increasingly, graduates are required to be more experienced and up to date with the latest technologies, processes and methodologies for their future career. Universities make a continuous effort (although not always possible) to provide their students with the best and most updated processes for their working life. And this requires continuous work and updating by professors and institutions. This has a special emphasis on the IT sector, which is currently in continuous evolution and where keeping up to date with the latest technologies is becoming highly complicated.

Another of the main problems that students may experience is that despite knowing a number of methods and technologies, they might have problems with the application of those in final products or services. In (Duderstadt, 2010), it is argued that technical skills may not be sufficient to translate intellectual knowledge into functional products and services. This may be due to a weak interconnection of technological skills to be applied in a final product. In this 
context, project-based learning (PBL) (Gary, 2015) is a methodology that has been very successful over the years in bringing students closer to real application cases.

PBL usually involves students in real situations where they have to apply the knowledge they have seen. This can lead to the realization of a product for an end-user, or the resolution of a real problem in a controlled environment. The projects developed are usually of a certain size and are usually developed in work groups, which favors the team and social skills of the students. In addition, this methodology allows students to deepen and reinforce their knowledge beyond that developed in class, where the contents are limited and it is not always possible to deepen everything the instructor desires. For example, in (Barak and Yehuditz, 2005) PBL is applied to enhance student's chemistry understanding. Furthermore, these projects can promote the active participation of students and motivate them to learn (GómezPablos, 2017).

Peer review (Topping, 2000) has also been successfully applied to improve the learning process of students. Peer review is a collaborative learning methodology where students assess other student's work providing their own feedback. This can lead to a better understanding of the peer's work since it exposes students to other types of solutions for a problem (which may be different from their own). Therefore, this process is enriching for students' learning in a course. Since students have to evaluate their peers, this also changes the role of the student-instructor (also known as flipped classroom) and often motivates students to complete their assignments. In addition, peer review allows students to develop their critical thinking skills. In (Conde et al., 2017), peer review is applied to technological courses to help students in technical studies to develop specific abilities such as getting more involved and critical thinking.

In this work, PBL is combined with peer review to improve the students' learning process with a special focus on their employability. On the one hand, the PBL approach aims to bring students closer to the real world by developing a solution to meet a need. On the other hand, with the application of peer review it is intended that students improve their critical skills and enhance their knowledge of the course by reviewing peer's work (and possibly other approaches). This project has been applied in a programming course at the University of Malaga, with the participation of 81 students and with the overarching goal of developing an Android application.

The projects developed were carried out by groups of 2-3 students and whose topic had to be agreed upon with the instructor. From the very beginning, we thought about the employability of the students, so in addition to the team, technical and critical skills acquired with the application of the mentioned approaches, students were invited to upload their projects to GitHub $^{1}$. GitHub is a widely used platform where software solutions and projects can be shared. It is also well-known in the industry sector, so the projects developed can serve the students as a demonstration of success for their future career. In addition, only a few weeks were previously dedicated to Android in the course, and thanks to the application of this approach students were able to delve deeper into the development of applications with this technology so in demand today.

1 https://github.com/ 


\section{Methodology}

This section presents a detailed description of how the peer review and PBL have been applied to final year students of a Software Engineering degree at the University of Malaga. The aim of this section is to show all the fundamental aspects of our experiment in a way that is fully reproducible by the reader.

A detailed description of the methodology and the different aspects of the application is given. This is followed by the objectives of the experiment and finally a discussion of the results according to the numbers obtained.

\subsection{Application}

In order to achieve the proposed objective, students carry out the design of their idea and the development of the Android app to implement it. Thus, in a transversal way aims to enrich the student's profile, especially with a view to immediate employability. Among candidates for new jobs in the fields of computer science and software engineering, it is a common practice to accompany the Curriculum Vitae with a portfolio of the candidate's software projects, mainly in Github. For this reason, this activity encourages students who do not yet have a personal Github to create one and those who already have one to create a new entry with this project.

For the application of peer review and PBL in a single activity, as applied in this course, groups of two students have been established to arbitrarily carry out the entire activity, thus encouraging both collaboration and cooperation in small, well-controlled groups. Once the groups are established and no one is left without a partner (in which case there would be a group of three students as a special case), a deadline of one week is proposed for the choice of a practice to be carried out. The instructor will have to approve the practice considering the originality of the ideas proposed, as well as the contribution to the resolution of real problems in society.

In spite of working with students of Software Engineering, who usually have developed skills for the use of technological tools, indeed in many occasions they do not get to know them in depth because they do not have the opportunity to do so. This activity provides an ideal frame as the groups should have scheduled virtual meetings in which they discuss the relevant topics of the project. In addition, there should be a shared repository between the group and the trainer in charge, in order to be able to carry out a follow-up.

Finally, although the application must be oriented to work on an Android device, any other framework can be used to provide specific functionalities for the project in question. As far as technical aspects are concerned, students are provided with a list of minimum requirements for their projects. Among them, it is described that the applications must be oriented to Android 10 (API level 29). At least three activities must be carried out, not limited to this figure, making use of the Singleton Map design pattern as a software engineering tool studied. Notification mechanisms and error information windows are to be used for end-user notification. It is essential to implement a database to connect to where all the information originated in the application is stored. The application must support multi-language (minimum Spanish and English).

The peer-review activity consists of a single activity that extends over 2 months of course period. 


\subsection{Rubrics}

It is considered essential that learners know in advance the items on which they will be assessed. Therefore, the instructor distributes a rubric. This rubric is specifically designed for this particular activity so that students are precisely informed about the task they are about to undertake. This rubric is included in the attached documentation for students. The process in which learners will be assessed by both the trainer and their peers is described very precisely. It also reveals the key points to be followed by the learner himself/herself when assessing the work of his/her peers.

Once the activities have been completed, the next step is the evaluation process. Each learner will evaluate the work of two of his/her peers, with the exception of his/her group partner. The results of these evaluations are reviewed by the instructor at the end of this phase. If the scores of the students are quite different from the score given by the instructor, these values will be discarded. In order to avoid possible external influences, such as friendship, enmity, etc.

It is important to mention the fact that all the data collected have been subjected to a process of anonymization in compliance with current privacy regulations. The $\mathrm{R}$ software tool has been used together with several statistical tools to process the results. As a consequence of the potential of this tool, graphs have been obtained that show significantly how the premises defined in our thesis are fulfilled in a tangible way within the imposed limitations. We must be critical in all aspects, first of all the students, although they have a similar profile, are dissimilar sets of individuals in the courses studied.

\subsection{Objectives \& Motivation}

The presented study has been developed in the framework of a Teaching Innovation Project PIE-(PIE19-209) ${ }^{2}$ funded by the University of Málaga.

This Educational Innovation project is carried out by a group of teaching assistants from different STEM departments of the University of Malaga who have come together with a common goal, once different weaknesses in specific learning points considered essential in the teaching activity have been recognized.

The work presented in this article is a practical application of the methodology detailed in (Serrano-Aguilera, 2021). Prior to the materialization of the project, a period of research and documentation of different methodologies was carried out. After which the peer review approach was identified as a very useful tool to work on in our courses, with a gradual and consensual adaptation. It is important to note that the project achieved is from an open call of the University of Malaga itself and that after its evaluation obtained the positive assessment by the evaluation committee, in September 2019, the implementation of the project began. Although the official start of the project is September 2019, the previous work had been carried out since or early January 2018.

The initial motivation for this project arose from the recurrent difficulty of a considerable part of the student body in acquiring the necessary skills in certain courses. Among these, it is worth highlighting the deficiencies shown in a high percentage of students in training related to employability.

2 https://www.uma.es/formacion/noticias/proyectos-de-innovacion-educativa-2019-2021/ 
In this context, PBL and peer review could act as a reinforcement activity that can improve the way to achieve the objectives. First of all, the designed activities aim at fostering the development of skills for this purpose as mentioned above. Secondly, the peer evaluation process allows for the development of critical analysis and the identification of errors. These aspects have led us to be aware of the enriching nature of the methodology by offering new perspectives that might not have been considered.

Therefore, we strongly believe that this methodology can be of great help to students, both in arriving at different solutions and in understanding the existence of multiple valid perspectives to address the challenges posed. This is because the pupils not only know their own project, but also that of their classmates. And by having to give detailed marks for them, they have to assess the work done.

\subsection{Approach}

This section details the context in which the peer review was applied. The methodology was applied in the course "Software Development for Embedded and Mobile Devices", a course from the last year of the Software Engineering degree at the University of Malaga. It was applied for the first time in the 2020-2021 academic year with a total of 81 students. The same activity was developed in the course 2018-2019 and 2019-2020 without the application of peer review with a total of 77 and 65 students respectively. This course is fundamentally practical, where students learn to get started with Arduino, Android programming and the programming of concurrency systems.

The application of peer review and PBL has been adapted to the peculiarities of this course. For this reason, it was decided to apply it in the part focused on Android. For the development of this part, we do not have many practical sessions to deepen the course, despite being one of the most demanded technologies for software development today and the future employment of students. This will allow students to go deeper into Android development thanks to the motivation that their work will be reviewed by other peers. In addition, as mentioned above, from the very beginning it was emphasized that the work would be available on GitHub so that this would serve as a showcase for their future career.

For the development of the activity, we used the "workshop" tool of our virtual campus. This tool allows students to upload their work, assign work among peers and make an evaluation with all the grades in the same tool, so it was very useful for the development of this activity. The rubric used and the practice requirements were available to the students from the very beginning. Then they were free to make groups among themselves, and the instructor individually solved the problems of the unpaired students. The workshop activity consists of 3 main parts: the submission phase, where students can submit their work to be evaluated; the evaluation phase, where students evaluate the work of two other peers; and finally, the evaluation phase where the instructor reviews all the grades and also grades the submitted work. In the evaluation phase, students had to select the rubric options that best matched the work they were reviewing. The instructor also performed the same operation as the students. For the first phase the students had about two months, 1 week for the peer evaluation phase, and the instructor reviewed all the works and evaluated them in 1 week.

Finally, the student's grades were a combination of $50 \%$ of the grades received among their peers (discarding borderline grades) and $50 \%$ of the grade received by the instructor. With the results obtained in the peer review application, we compared the results with the previous academic years (2018-2019 and 2019-2020) where the same activity was done, to assess whether it improves the students' performance. As a general note, the resulting applications 
were of high quality, including an application for geolocation in public places to avoid crowds of people in this COVID19 season; an application to improve the animal adoption process, and a game to guess songs from small fragments of them.

\section{Results}

Once the activity has been carried out and the grades have been obtained anonymously, we will analyze them and compare them with the results obtained in previous academic years to see the feasibility of the application of this approach in this context. The results follow the Spanish 010 grading scale, with 0 being the lowest and 10 the highest. The intervals 0-5 (fail), 5-7 (pass), 7-9 (good) and 9-10 (outstanding) were used to compare the students' grades. Figures 1 and 2 show the grades and the number of students and the percentage of students in those intervals, respectively.

Regarding the grades and intervals (Figure 1), it can be observed that in the 2020-2021 academic course with the application of the peer review and PBL the number of students with a grade of good has increased considerably. The number of students with outstanding grades has also decreased with respect to the previous year. However, one of the main advantages of the application of this approach is the notable reduction in the number of students who did not pass with respect to previous academic years.

Figure 1: Number of students and grades of the proposed activity. Course 2020-2021 with peer review

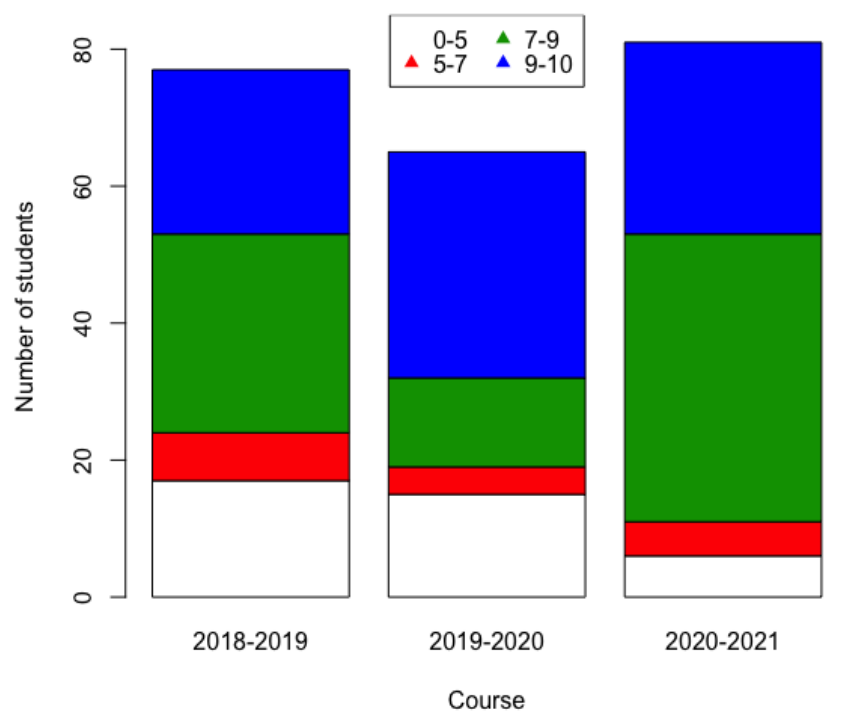

Regarding the percentage of grades (Figure 2), we can corroborate what we have discussed with the total number of students. The percentage of students during the course with the application of peer review and PBL with a grade of 7-9 has increased considerably while the number of students who did not pass the activity has decreased notably. 
Figure 2: Percentage of students and grades of the proposed activity. Course 2020-2021 with peer review

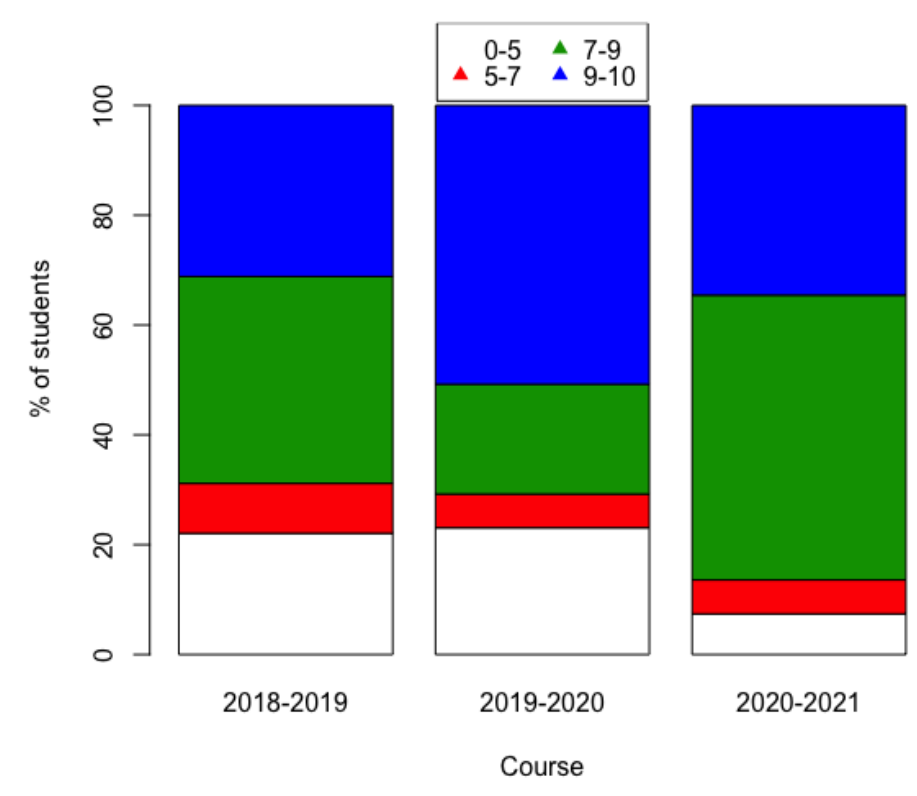

\subsection{Statistical results}

Statistical analysis was also carried out to analyze the overall grades of the three courses studied, as shown in Figure 3. Each block indicates the confidence interval between 25 and $75 \%$ of the data, the red asterisks denote the mean, and the black stripes within the block denote the median. As can be seen, the confidence interval has narrowed in the 2020-2021 course, which denotes that the grades are not so dispersed. In addition, the mean grade is more than one point higher compared to previous years, where it is very similar. With this we demonstrate that with the application of this approach the overall grades of the students have increased and the percentage of failed students in this activity has been reduced considerably.

Figure 3: Median, mean and confidence intervals of the proposed activity. Course 2020-2021 with peer review

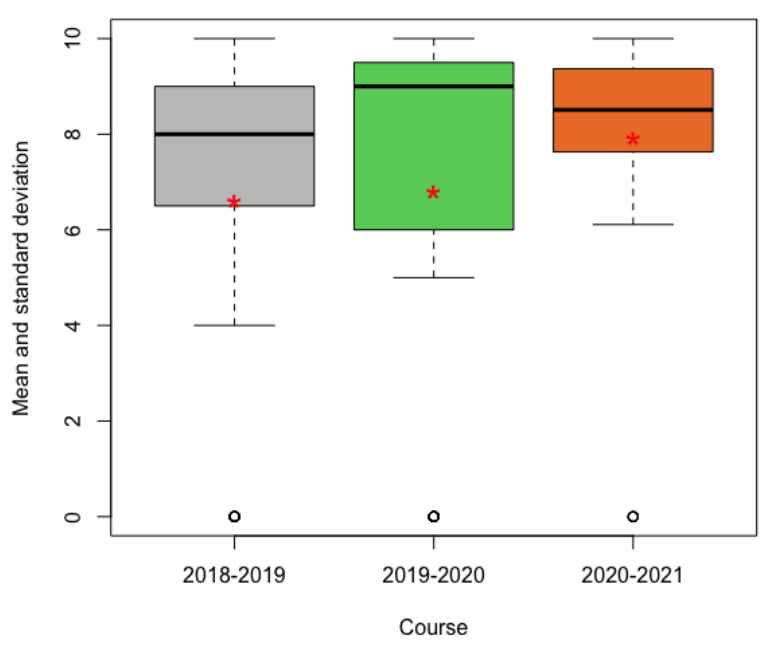




\subsection{Students' feedback}

Finally, we sent a survey to the students after the activity to assess whether they were able to improve their knowledge and their level of motivation with respect to the activity. The survey was completed by 37 students out of 81 . Figures 4 and 5 show respectively the degree of knowledge acquired and the level of motivation on a scale of 0-10. Regarding the level of knowledge acquired, $73 \%$ of the students indicated with a value greater than or equal to 8 that with this activity they improved the knowledge acquired in this course. Regarding motivation, $67.5 \%$ of the students indicated with a value greater than or equal to 8 that their motivation had increased with this activity, and $83.8 \%$ with a value greater than or equal to 7 . We, therefore, consider these results positive and demonstrate how in general this activity has contributed to improving the knowledge and motivation of the students.

Figure 4: Student's feedback on knowledge reinforcement with this activity

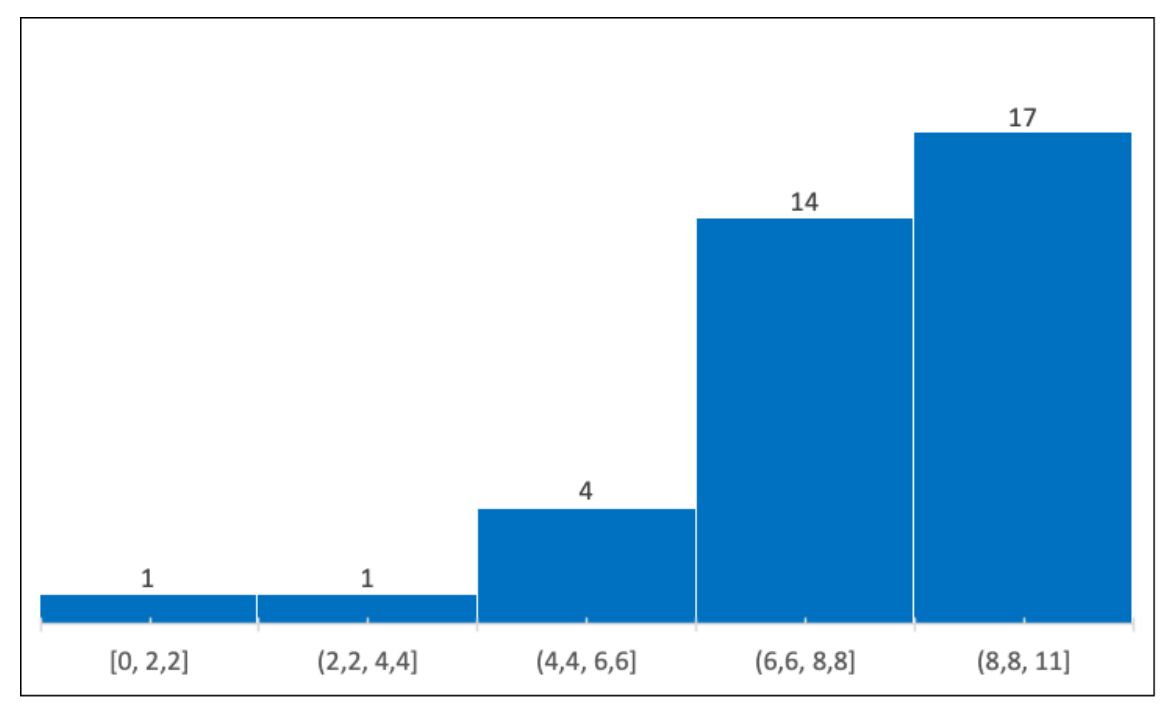

Figure 5: Student's feedback on motivation with this activity

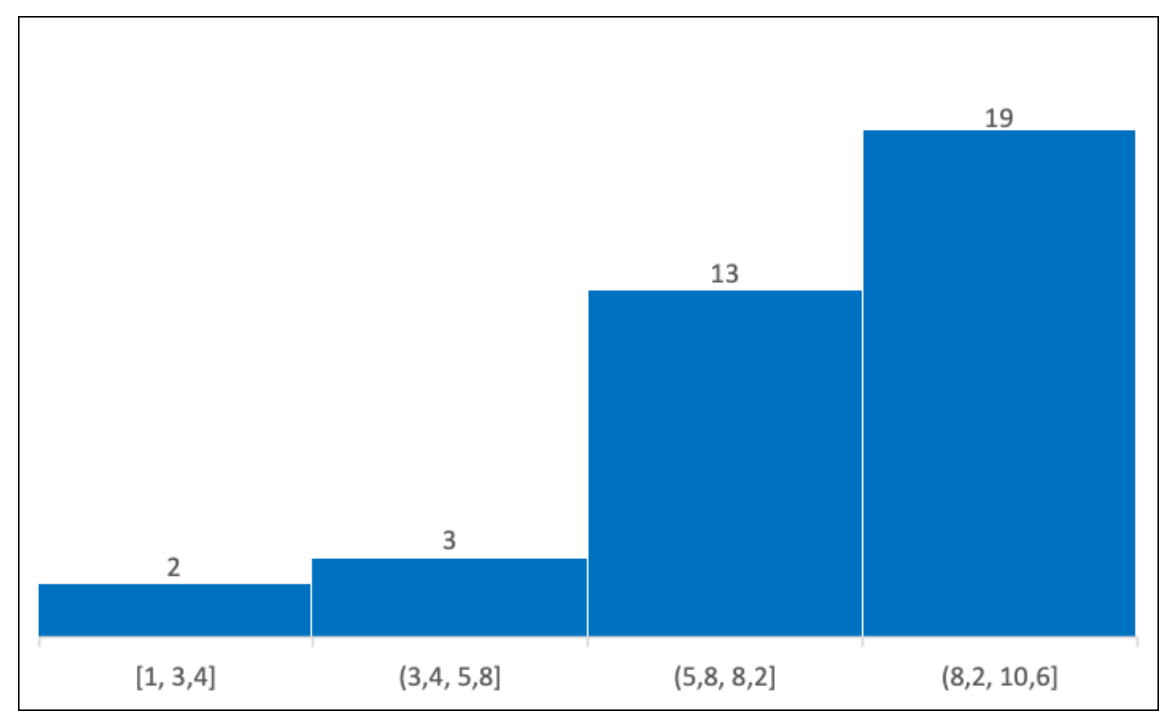




\section{Conclusion}

In this article, the application of an experience based on peer review and PBL has been presented. This experience has been developed in an IT course with 81 students participating at the University of Malaga, whose objective was the development of an Android application. The overarching objective of this experience has been to contribute to improving the employability of students in their future work, exposing them to a real problem to be solved. In addition, this has also contributed to improving their critical skills, reinforcing group work and allowing them to evaluate and learn from the work of their peers. The analyzed results show an improvement in performance compared to the same activity in previous years where this approach was not applied. The motivation and acquisition of knowledge by the students in the course showed positive results with the developed activity.

As future work, we propose to continue this study for a number of consecutive years. In order to be able to extract less biased information and to be able to reaffirm or refute the conclusions drawn from these experiences. We are also working on a refinement of the rubrics provided with the results of the experiences and student feedback. This is collected through satisfaction surveys at the end of the academic year. These surveys also offer us the possibility of exploring the degree of satisfaction that the application of these methodologies has among them.

\section{Acknowledgment}

This paper is an output of the Spanish Teaching Innovation Project PIE19-209 ("Collaborative evaluation to strengthen student competencies through an active methodology from the teaching and learning process to evaluation") at the University of Malaga.

\section{References}

Barak, Miri, and Yehudit Judy Dori. (2005) "Enhancing undergraduate students' chemistry understanding through project- based learning in an IT environment." Science education vol 89, no 1, pp. 117-139.

Conde, M. Á., et al. (2017) "Application of peer review techniques in engineering education." International Journal of Engineering Education, vol 33, no 2, pp. 918-926.

Duderstadt, J. J. (2010). "Engineering for a changing world." In Holistic engineering education. Springer, New York, NY., pp. 17-35.

Gary, K. (2015). “Project-based learning.” Computer, vol. 48, no 2, pp. 98-100.

Gómez-Pablos, Verónica Basilotta, Marta Martín del Pozo, and Ana García-Valcárcel MuñozRepiso. (2017) "Project-based learning (PBL) through the incorporation of digital technologies: An evaluation based on the experience of serving teachers." Computers in Human Behavior, vol 68, pp. 501-512.

Serrano-Aguilera, Juan Jose, et al. (2021) "Using Peer Review for Student Performance Enhancement: Experiences in a Multidisciplinary Higher Education Setting." Education Sciences, vol. 11, no 2, p. 71. 
3rd International Academic Conference on Education, Teaching \& Learning May 21-23, 2021

Topping, K. J., Smith, E. F., Swanson, I., \& Elliot, A. (2000). "Formative peer assessment of academic writing between postgraduate students." Assessment \& evaluation in higher education, vol 25, no 2, pp. 149-169. 